\title{
VARIAÇÃO SAZONAL NA DIETA DE Trinomys albispinus (RODENTIA, ECHIMYIDAE) EM UMA ÁREA DE CAMPOS RUPESTRES, NO SUDESTE DO BRASIL
}

\author{
Verônica Guedes Sena ${ }^{1}$ \& Leonardo Guimarães Lessa ${ }^{1,2 *}$ \\ ${ }^{1}$ Universidade Federal dos Vales do Jequitinhonha e Mucuri, Programa de Pós-Graduação em Biologia Animal. Rodovia \\ MGT 367, n5000. Diamantina. CEP: 39100-000. MG, Brasil. \\ ${ }^{2}$ Universidade Federal dos Vales do Jequitinhonha e Mucuri, Departamento. de Ciências Biológicas, Laboratório de \\ Ecologia, Rodovia MGT 367, n 5000. Diamantina. CEP: 39100-000. MG, Brasil. \\ E-mails: vsena1994@gmail.com; leoglessa@gmail.com (*autor correspondente)
}

\begin{abstract}
Resumo: O gênero Trinomys (Echimyidae) é composto por espécies de hábitos noturnos, terrícolas, e com ocorrência, em sua maioria, em áreas de Mata Atlântica no Brasil. Entretanto, a espécie de rato-de-espinho Trinomys albispinus (I. Geoffroy St.-Hilaire, 1838) apresenta distribuição por biomas savânicos brasileiros como a Caatinga e o Cerrado. Apesar dessa inusitada adaptação para ambientes secos, pouco se sabe sobre a ecologia alimentar de T. albispinus ao longo de sua área de distribuição. O presente estudo teve como objetivo descrever a dieta de T. albispinus e avaliar sua variação sazonal e intersexual. O estudo foi desenvolvido em área de campo rupestre, localizada na porção meridional da Cadeia do Espinhaço, Brasil, no período de fevereiro a dezembro de 2014. Foram analisadas 41 amostras fecais, sendo observada uma dieta diversificada composta principalmente por artrópodes (90\%), partes vegetativas de plantas (85\%), frutos $(27 \%)$ e vertebrados (2 \%). O roedor T. albispinus apresentou variação sazonal (seca versus chuva) e intersexual (machos versus fêmeas) na composição da dieta. Esses resultados podem estar relacionados com as diferenças na demanda energética entre machos e fêmeas ao longo do ano em função do ciclo reprodutivo. Este é o primeiro estudo específico sobre a dieta de T. albispinus em campos rupestres e o primeiro registro da possível predação de lagartos por roedores da família Echimyidae.
\end{abstract}

Palavras chave: Cerrado; Echimyidae; Ecologia alimentar; Mammalia; Neotrópicos.

SEASONAL VARIATION IN THE DIET OF TRINOMYS ALBISPINUS (RODENTIA, ECHIMYIDAE) IN A RUPESTRIAN GRASSLAND AREA, SOUTHEASTERN BRAZIL. The species of spiny-rats of the genus Trinomys (Echimyidae) demonstrates nocturnal and terrestrial habitats and they are distributed mostly in the Atlantic forests of Brazil. However, Trinomys albispinus (I. Geoffroy St.-Hilaire, 1838) is distributed throughout the Brazilian savanna biomes of Caatinga and Cerrado. Despite this unusual adaptation to dry environments, few is known about the feeding ecology of this species along its range of distribution. The present study aimed to describe the diet of the spiny-rat Trinomys albispinus (I. Geoffroy St.-Hilaire, 1838) and to evaluate its seasonal and intraspecific variation. The study was carried out in a rupestrian grassland area located in the Southern portion of the Cadeia do Espinhaço, Brazil, from February to December 2014. Forty-one fecal samples were analyzed and a diversified diet consisting mainly of arthropods (90\%), vegetative parts of plants (85\%), fruits (27\%) and vertebrates (2\%). Trinomys albispinus showed seasonal (dry versus rain) and intraspecific (male versus female) variation in diet composition. These results may be related to differences in energy requirements between males and females throughout the year as a function of the reproductive 
cycle. This is the first diet study of T. albispinus in the rupestrian grasslands and the first record of possible predation of lizards by rodents of the family Echimyidae.

Keywords: Cerrado; Echimyidae; feeding ecology; Mammalia; neotropics.

\section{INTRODUÇÃO}

Roedores neotropicais da família Echimyidae (Proechimys spp., Thrichomys spp. e Trinomys spp.) têm sido tradicionalmente considerados como espécies de hábitos alimentares frugívoro/ herbívoro ou frugívoro/granívoro (Paglia et al. 2012). Entretanto, a despeito de sua importância, informações relativas à ecologia alimentar e outros aspectos relacionados à história natural de roedores do gênero Trinomys são ainda escassas (Patton et al. 2015), e um mesmo padrão de dieta é estendido para todas as espécies do gênero (Paglia et al.2012). Estudos de dieta são de primordialimportância para o entendimento da biologia de qualquer espécie nos níveis individual e populacional (Martins et al. 2006), contribuindo para a compreensão de importantes aspectos ecológicos como o uso do hábitat (Albanese et al. 2012), seleção de recursos (Pinotti et al. 2011, Melo et al. 2018), predação e/ ou dispersão de sementes (Lessa \& Costa 2009, Lessa et al. 2013, Sahley et al. 2016) e estrutura da comunidade (Lessa \& Paula 2014).

Um importante aspecto a se considerar na avaliação da dieta deuma espécieéa disponibilidade dos recursos alimentares (Pinotti et al. 2011). No Cerrado, onde a variação sazonal dos recursos é determinada pela precipitação (Murphy \& Lugo 1986), a variação na oferta de alimentos pode ocasionar flutuação das populações e variações em sua atividade reprodutiva (Adler 1998, Mattos 2014). Neste sentido, ambientes sazonais como os campos rupestres no bioma Cerrado, podem influenciar a dieta das espécies, uma vez que, a disponibilidade de recursos, especialmente artrópodes e frutos, pode variar durante o ano (Mantovani \& Martins 1988, Pinheiro et al. 2002, Pinotti et al. 2011). Além disso, pequenos mamíferos podem apresentar variações intraespecíficas na composição da dieta em função de diferentes fatores como sazonalidade, idade, condição reprodutiva individual e sexo (Martins et al. 2006, Camargo et al. 2014, Melo et al. 2018). As escassas informações disponíveis sobre a ecologia de Trinomys sugerem que as espécies desse gênero podem se reproduzir ao longo de todo o ano, porém, apresentando um pico reprodutivo durante a estação chuvosa, provavelmente a fim de ajustar a lactação a um período mais favorável com relação ao suprimento de recursos (Bergallo \& Magnusson 1999, Patton et al. 2015).

O gênero Trinomys Thomas, 1921, é composto por espécies de hábitos noturnos, terrícolas, e com ocorrência, em sua maioria, em áreas de Mata Atlântica no Brasil (Paglia et al. 2012, Patton et al. 2015). Entretanto, a espécie de rato-de-espinho Trinomys albispinus (I. Geoffroy St.-Hilaire, 1838), apresenta distribuição por biomas savânicos brasileiros como a Caatinga e o Cerrado (Paglia et al. 2012, Patton et al. 2015), onde pode ocorrer em áreas de campos rupestres caracterizadas por afloramentos rochosos de quartzito e gramíneas ao longo da Cadeia do Espinhaço (Lessa et al. 2008, Patton et al. 2015). Os ratos-de-espinho são pequenos roedores (120 - 230 g) solitários e são considerados como uma das formas mais especializadas do gênero Trinomys, apresentando adaptações para ambientes secos (Patton et al. 2015).

Até o presente momento, não se conhecem estudos específicos sobre a ecologia alimentar de T. albispinus ao longo de sua área de distribuição. Neste sentido, o presente estudo pretende descrever a composição da dieta de T. albispinus, a partir de amostras fecais coletadas em uma área de campos rupestres na porção meridional da Cadeia do Espinhaço, sudeste do Brasil. Especificamente, pretendemos investigar a variação sazonal (seca versus chuva) e intersexual (machos versus fêmeas) na dieta da espécie. Considerando que os campos rupestres apresentam marcada sazonalidade (Silveira et al. 2016), o que potencialmente afeta a disponibilidade de recursos, esperamos encontrar variação sazonal na dieta da espécie ao longo do ano com uma dieta menos diversa na estação chuvosa e mais diversa na estação seca (Mattos 2014). Apesar das informações preliminares sugerirem a ausência de sazonalidade na reprodução de $T$. albispinus (Bergallo \& Magnusson 1999, Patton et al. 2015), esperamos que a dieta varie entre os sexos, considerando a demanda energética de machos 
e fêmeas durante o ciclo reprodutivo (Bergallo \& Magnusson 1999).

\section{MATERIAL E MÉTODOS}

\section{Área de estudo}

Realizamos este estudo no Parque Nacional das Sempre Vivas (PARNA SV, 1754'50.48" S, $43^{\circ} 47^{\prime} 32.60^{\prime \prime} \mathrm{W}$ ), localizado na porção meridional da Cadeia do Espinhaço, Minas Gerais, Brasil (Figura 1). O PARNA SV possui uma área aproximada de 124 mil hectares e abriga um complexo de fitofisionomias característica do bioma Cerrado como: campos limpos, campos rupestres, Cerrado stricto sensu e fragmentos de florestas decíduas e semidecíduas (Sano et al. 2007). O clima é definido como tipo Cwb, na classificação de Köppen, com invernos secos (abril a setembro) e verões úmidos (outubro a março) (Alvares etal.2013). A temperatura média é de $20^{\circ} \mathrm{C}$, a precipitação anual varia entre 1,250 e 1,500 mm e a altitude varia de 650 a 1,520 m (Leal et al. 2008).
Os espécimes foram coletados em uma área de campos rupestres intercalada por capões de mata localizada na porção sul do Parque. A formação de campos rupestres é caracterizada como um mosaico de vegetação endêmico do Brasil, associada a afloramentos rochosos de quartzito, arenito ou ferro, ao longo das terras altas (acima de $900 \mathrm{~m}$ ) na Cadeia do Espinhaço, nos estados da Bahia e Minas Gerais, Brasil (Alves et al. 2014, Silveira et al. 2016). A despeito de sua importância, o conhecimento relativo à ecologia, composição e distribuição da fauna de vertebrados que habitam estas áreas montanhosas é ainda incipiente (Alves et al. 2014).

\section{Composição da dieta}

Analisamos 41 amostras fecais de T. albispinus coletadas nos meses de fevereiro, abril, junho, agosto, outubro e dezembro de 2014. Todos os roedores capturados foram marcados com anilhas numeradas (Zootech ${ }^{\circledR}$ ) e soltos no mesmo local de captura. As fezes foram coletadas de

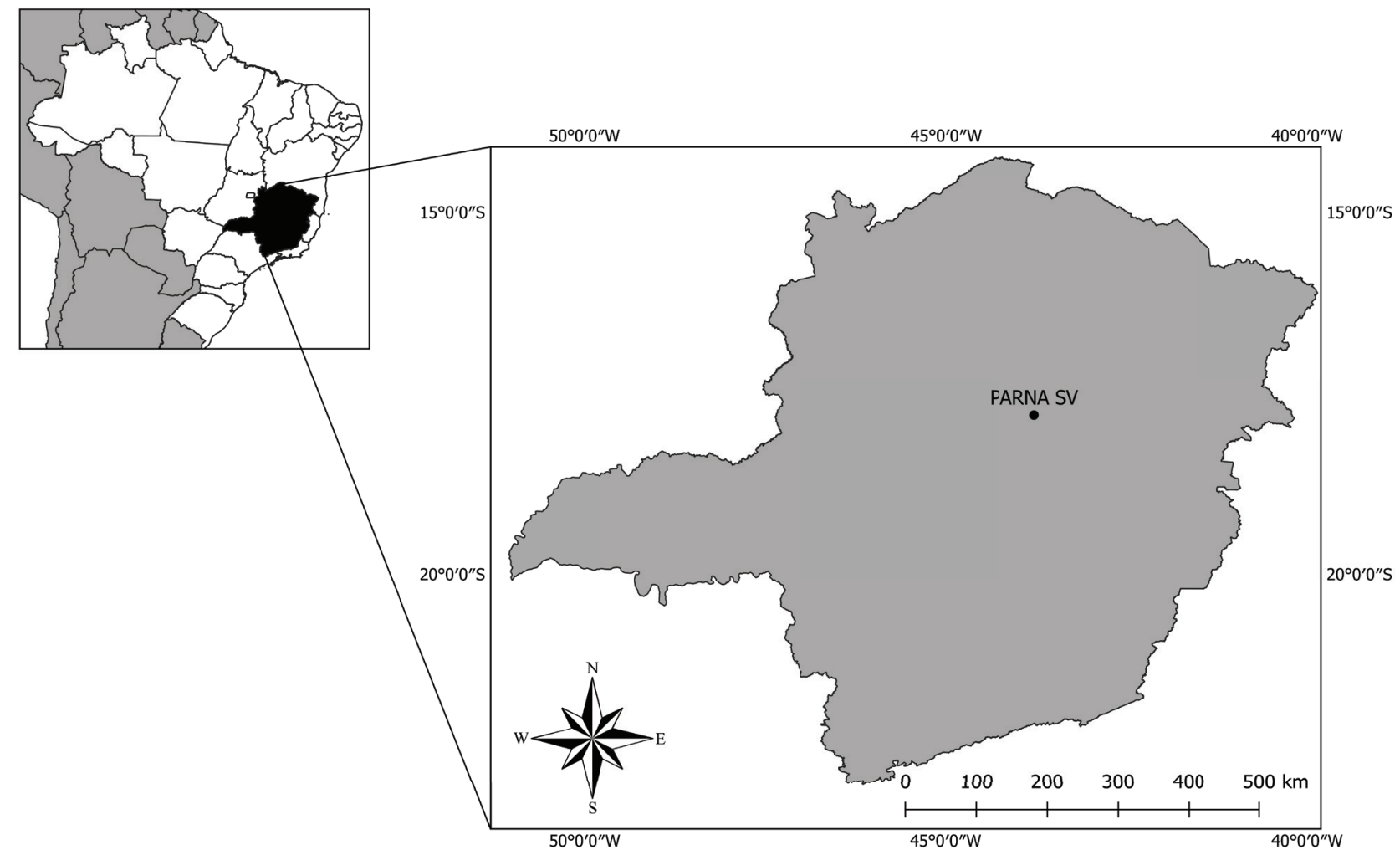

Figura 1. Área de estudo, localizada no do Parque Nacional das Sempre Vivas (PARNA SV), Minas Gerais, Brasil.

Figure 1. Study area located at the Parque Nacional das Sempre Vivas (PARNA SV), Minas Gerais, Brazil. 
cada espécime durante o procedimento de manipulação ou no interior das armadilhas. As fezes coletadas foram identificadas e armazenadas em recipientes plásticos secos e mantidas sob refrigeração para evitar a ação de fungos. Para cada amostra fecal foram registradas informações referentes à: i) local de coleta; ii) data; iii) número da anilha; iv) sexo do indivíduo. Em laboratório, o material foi lavado em água destilada com o auxílio de peneira granulométrica com malha metálica de 0,1 mm. Em seguida, com o auxílio de um microscópio estereoscópico, todos os itens alimentares detectados foram separados nas seguintes categorias: i) sementes; ii) flores; iii) partes vegetativas de plantas (folhas e caules); iv) artrópodes; v) outros.

Identificamos os itens coletados até a menor categoria taxonômica possível. Identificamos os invertebrados com o auxílio de literatura especializada (Triplehorn \& Johnson, 2011, Constantino, 2012, Constantino, 2015) e através da comparação com coleção de referência de artrópodes coletados na mesma área do presente estudo (coleção depositada no Laboratório de Ecologia da Universidade Federal dos Vales do Jequitinhonha e Mucuri). Identificamos as escamas de lagartos encontradas nas amostras fecais através de análise morfológica (número e posição dos mucros e aberturas sensoriais) e morfométricas (comprimento total, largura total, comprimento do mucro e largura mucro-base). Posteriormente, comparamos com as informações disponíveis na literatura especializada (Franco \& Ferreira 2002, Franco et al. 2017).

Os espécimes de T. albispinus foram identificados por comparação com material depositado em coleções científicas (Coleção de pequenos mamíferos da UFVJM e Coleção de Mastozoologia do Museu de Ciências Naturais da PUC-MINAS) e descrição na literatura especializada (Patton et al. 2015). O estudo foi devidamente licenciado junto ao Sistema de Autorização e Informação da Biodiversidade (SISBIO, licença número 52836-1).

\section{Análises estatísticas}

Utilizamos a Frequência Relativa de Ocorrência (FRO \%) para determinar a contribuição relativa de cada item na dieta de T. albispinus. A FRO é expressa como o número amostras em que um item é registrado (n), dividido pelo número total de amostras analisadas $(\mathrm{N})$, e multiplicadas por 100 (Korschgen 1987). Utilizamos o teste-G para comparar as diferenças na proporção de itens alimentares consumidos (FRO \%) entre: estações (seca e chuva) e entre sexos (machos e fêmeas). Para análise da diversidade da dieta utilizamos o índice de diversidade de Shannon-Wiener ( $\left.\mathrm{H}^{\prime}\right)$. O valor do índice foi comparado utilizando o teste $t$ de Hutcheson (Zar 2010). Para todas as análises excluímos as amostras de recapturas dos mesmos indivíduos em dias consecutivos a fim de evitar pseudoreplicação (Hurlbert 1984). Para executar o teste-G utilizamos o software BioEstat ${ }^{\circledR} 5.3$ (Ayres et al. 2007) e para o teste $t$ utilizamos o software PAST® 3.15 (Hammer et al. 2001).

\section{RESULTADOS}

Analisamos 41 amostras fecais de T. albispinus, sendo: 30 amostras coletadas na estação seca e 11 na estação chuvosa; 27 amostras de indivíduos machos e 14 amostras de fêmeas. Foram identificados nas amostras fecais: material vegetativo (folhas e fragmentos de caules), artrópodes, flores, frutos, sementes e um pequeno vertebrado (Tabela 1).

Registramos artrópodes em 90 \% das amostras, sendo a ordem Hymenoptera observada com maior frequência ( $\mathrm{FRO}=85 \%$ ), seguida pela ordem Coleoptera $(\mathrm{FRO}=27 \%)$. As ordens Isoptera, Lepidoptera, Psicoptera, Protura, Diptera, Diplura e Orthoptera foram registrados em frequências abaixo de $12 \%$. Observamos também a presença de escamas de lagartos (Tropiduridae) em uma única amostra pertencente a um indivíduo do sexo feminino. Material vegetativo foi a segunda categoria alimentar mais frequente nas amostras $($ FRO $=85 \%)$, seguido por sementes e flores, enquanto fragmentos de frutos (pericarpo) foram observados em baixa frequência (FRO $=10 \%$; Tabela 1).

Observamos diferença significativa na frequência dos itens alimentares presentes nas amostras entre as estações $(\mathrm{G}=102,546 ; \mathrm{gl}=$ 14; $\mathrm{p}<0,0001)$, e entre os sexos $(\mathrm{G}=81,346 ; \mathrm{gl}=$ 14; $\mathrm{p}<0,0001)$. Observamos também diferença significativa na diversidade dos itens consumidos entre as estações (H'seca $=2,268$; H'chuvosa $=1,524$; $\mathrm{t}=6,712 ; \mathrm{gl}=562,31 ; \mathrm{p}<0,0001)$. Alguns artrópodes das ordens Diptera, Diplura, Orthoptera, Protura, 
Tabela 1. Frequência Relativa de Ocorrência (\%) dos itens alimentares identificados nas amostras fecais de Trinomys albispinus (estações seca e chuvosa; machos e fêmeas) em uma área de campos rupestres, Minas Gerais, Brasil. $\mathrm{N}=$ número de amostras

Table 1. Relative Frequency of Occurrence (\%) of food items identified in the scats of Trinomys albispinus (dry and rainy seasons; males and females) in a rock grassland area, Minas Gerais, Brazil. $N=$ number of samples.

\begin{tabular}{|c|c|c|c|c|c|}
\hline $\begin{array}{l}\text { Categorias } \\
\text { alimentares }\end{array}$ & $\begin{array}{c}\text { Seca } \\
(\mathrm{N}=30)\end{array}$ & $\begin{array}{l}\text { Chuva } \\
(\mathrm{N}=11)\end{array}$ & $\begin{array}{l}\text { Machos } \\
(\mathrm{N}=27)\end{array}$ & $\begin{array}{l}\text { Fêmeas } \\
(\mathrm{N}=14)\end{array}$ & $\begin{array}{c}\text { Total } \\
(\mathrm{N}=41)\end{array}$ \\
\hline Sementes & 27 & 27 & 30 & 21 & 27 \\
\hline Melastomataceae & - & 9 & 4 & - & 2 \\
\hline $\begin{array}{l}\text { Sementes não } \\
\text { identificadas }\end{array}$ & 27 & 18 & 26 & 21 & 24 \\
\hline $\begin{array}{l}\text { Frutos } \\
\text { (fragmentos) }\end{array}$ & 10 & 9 & 7 & 14 & 10 \\
\hline Flores & 17 & 36 & 19 & 29 & 22 \\
\hline $\begin{array}{l}\text { Material } \\
\text { Vegetativo }\end{array}$ & 80 & 100 & 89 & 79 & 85 \\
\hline Artrópodes & 90 & 90 & 85 & 100 & 90 \\
\hline Orthoptera & 3 & - & 4 & - & 2 \\
\hline Diptera & 3 & - & - & 7 & 2 \\
\hline Protura & 3 & - & - & 7 & 2 \\
\hline Psicoptera & 3 & - & 4 & - & 2 \\
\hline Lepidoptera & 3 & - & - & 7 & 2 \\
\hline Diplura & 10 & - & 11 & - & 7 \\
\hline Isoptera & 17 & - & 15 & 7 & 12 \\
\hline Coleoptera & 33 & 9 & 22 & 36 & 27 \\
\hline Hymenoptera & 83 & 90 & 81 & 93 & 85 \\
\hline \multicolumn{6}{|l|}{ Vertebrados } \\
\hline $\begin{array}{l}\text { Lacertilia } \\
\text { (Tropiduridae) }\end{array}$ & 3 & - & - & 7 & 2 \\
\hline
\end{tabular}

Psicoptera e Lepidoptera foram consumidos apenas na estação seca, (Tabela 1). Não observamos diferença significativa na diversidade da dieta entre sexos (H'machos $=1,871 ;$ H'fêmeas $=1,957 ; t$ $=-1,019 ; \mathrm{gl}=577,39 ; \mathrm{p}=0,308)$, apesar de alguns artrópodes terem sido registrados em baixas frequências apenas nas amostras de machos (Orthoptera e Psicoptera) ou de fêmeas (Diptera, Protura e Lepidoptera).

\section{DISCUSSÃO}

De acordo com nossos resultados, a dieta de $T$. albispinusé composta principalmente por diversos grupos de artrópodes e partes vegetativas de plantas, enquanto frutos (sementes e fragmentos de pericarpo), flores e até mesmo escamas de lagarto foram registrados em baixa frequência nas amostras de fezes na área de estudo. Neste sentido, a classificação trófica para T. albispinus apresentada neste estudo difere da caracterização geral sugerida para Trinomysspp. (Paglia etal.2012), e não está de acordo com Emmons \& Feer (1997), que indica que os ratos-de-espinho (Trinomys spp. e Proechimys spp.) consomem principalmente frutos e fungos, raramente consumindo insetos e partes vegetativas de plantas (folhas e caules). Portanto, sugerimos a reclassificação desse roedor para insetívoro/onívoro, e não como uma espécie primariamente frugívora (ver Emmons \& Feer 1997, Paglia et al. 2012). Observamos que T. albispinus apresentou variação sazonal 
na composição da dieta, consumindo partes vegetativas de plantas ao longo de todo o ano, mas incorporando diferentes grupos de artrópodes principalmente durante a estação seca. Segundo Talamoni et al. 2008, o consumo relativamente alto de artrópodes por pequenos roedores (Echimyidae e Cricetidae) durante a estação seca pode indicar a busca ativa deste recurso. Esse consumo diferencial de artrópodes entre estações foi também registrado em uma área de Cerrado na dieta do roedor equimídeo T. apereoides (Lessa \& Costa 2009), e em uma área de campos rupestres na dieta do pequeno marsupial terrícola Monodelphis domestica (Carvalho et al. 2019). Estudos recentes sobre os hábitos alimentares de pequenos mamíferos (Rodentia e Didelphimorphia) em formações abertas de Cerrado (como a área de campo rupestre investigada) tem evidenciado um padrão oportunista de forrageamento, onde as espécies alteram sua dieta em função da disponibilidade sazonal de recursos (ver Lessa \& Costa 2009, Cantor etal.2013, Lessa etal.2013, Melo et al. 2018, Carvalho et al. 2019). Entretanto, outros estudos registraram um padrão seletivo, onde os recursos são consumidos independentemente da disponibilidade local (ver Pinotti et al. 2011, Camargo et al. 2014). Devido a essa variação nos padrões de forrageio e a ausência de dados sobre a disponibilidade de recursos em nossa área de estudo, nossa dedução com relação ao possível padrão de forrageamento oportunista por $T$. albispinus ainda é limitada. Porém, cabe ressaltar que no bioma Cerrado, Hymenoptera, Coleoptera e Isoptera compreendem as ordens mais comuns de insetos (Pinheiro et al. 2002). Da mesma maneira, partes vegetativas de plantas (folhas e caules) constituem um recurso disponível e acessível ao longo de todo o ano para diversas espécies de roedores (Vieira \& Briani 2013), sendo estes os recursos registrados com maior frequência na dieta de T. albispinus durante o presente estudo.

Outro resultado importante observado foi a diferença na composição da dieta entre machos e fêmeas. Nas savanas Neotropicais (como Cerrado e Caatinga), um pico reprodutivo para as fêmeas de T. albispinus parece ocorrer durante a estação chuvosa (Patton et al. 2015). Esse padrão pode estar relacionado com a maior disponibilidade de recursos (artrópodes e frutos) neste período (Mantovani \& Martins 1988,
Pinheiro et al. 2002). Mesmo para as espécies de roedores que se reproduzem ao longo de todo o ano, a intensidade da reprodução pode variar em resposta às condições ambientais (Bergallo 1994, Bergallo \& Magnusson 1999). Assim sendo, uma possível explicação para a variação observada na composição da dieta entre machos e fêmeas de T. albispinus em nossa área de estudo, pode ser um indicativo sobre as diferenças na demanda energética ao longo do ano em função do ciclo reprodutivo.

Cabe ressaltar que nossa investigação sobre a dieta de T.albispinus foi realizada através da análise de conteúdo fecal, o que gera a possibilidade de subestimar a proporção de itens facilmente digeríveis como tecidos moles de artrópodes e polpas de frutos (ver Dickman \& Huang 1988). Além disso, não podemos descartar a possibilidade de que as altas frequências de alguns grupos de artrópodes nas amostras (por exemplo, Hymenoptera e Coleoptera) possam refletir o fato de que suas estruturas quitinizadas facilitem sua preservação, tornando-as mais detectáveis nas amostras fecais. Roedores equimídeos são equipados com um eficiente aparato mastigatório composto por dentes incisivos em forma de cinzel, uma série de dentes molares trituradores e mandíbulas que podem se mover tanto lateralmente quanto para frente e para trás (Price \& Jenkins 1986). Este aparato mastigatório permite o eficiente processamento de diversos itens alimentares, como artrópodes, frutos e sementes (Lessa \& Costa 2009). Mesmo cientes destas limitações metodológicas, destacamos que, este é o primeiro estudo específico sobre a dieta natural de T. albispinus em campos rupestres e o primeiro registro da possível predação de lagartos por roedores Neotropicais da família Echimyidae.

\section{AGRADECIMENTOS}

Agradecemos a equipe do Laboratório de Ecologia e Conservação da Universidade Federal dos Vales do Jequitinhonha e Mucuri pelo auxílio durante as atividades de campo e ao Instituto Chico Mendes de Conservação da Biodiversidade (ICMBIO escritório Regional de Diamantina) pelo suporte logístico no PARNA Sempre Vivas. Agradecemos também à contribuição de dois revisores anônimos na melhoria do manuscrito. 


\section{REFERÊNCIAS}

Albanese, S., Dacar, M. A., \& Ojeda, R. A. 2012. Unvarying diet of a Neotropical desert marsupial inhabiting a variable environment: the case of Thylamys pallidior. Acta Theriologica, 57(2), 185-188. DOI: 10.1007/s13364-011-0057-6

Adler, G. H. 1998. Impact of resources abundance on populations of a tropical forest rodent. Ecology, 79(1), 242-254. DOI: http://doi.org/ck5t46.

Alvares, C. A., Stape, J. L., Sentelhas, P. C., de Moraes Gonçalves, J. L., \& Sparovek, G. 2013. Köppen's climate classification map for Brazil. Meteorologische Zeitschrift, 22(6), 711-728. DOI: 10.1127/0941-2948/2013/0507

Alves, R. J. V., Silva, N. V., Oliveira, J. A., \& Medeiros, D. 2014. Circumscribing campo rupestre megadiverse Brazilian rock montane savannas. Brazilian Journal of Biology, 74(2), 355-362. DOI: 10.1580/1519-6984.23212

Ayres, M., Ayres Junior, M., Ayres, D. L., \& Santos, A. S. 2007. Bioestat 5.0 - Aplicações estatísticas nas áreas das ciências biomédicas. Belém, PA: ONG Mamirauá: p. 364.

Bergallo, H. G. 1994. Ecology of a small mammal community in an Atlantic forest area in southeastern Brazil. Studies on Neotropical Fauna and Environment, 29(4), 197-217. DOI: $10.1080 / 01650529409360932$

Bergallo, H. G., \& Magnusson, W. E. 1999. Effects of climate and food availability on four rodent species in southeastern Brazil. Journal of Mammalogy, 80(2), 472-486. DOI: $10.2307 / 1383294$

Cantor, M., Pires, M. M., Longo, G. O., Guimarães, P. R., \& Setz, E. Z. F. 2013. Individual variation in resource use by opossums leading to nested fruit consumption. Oikos, 122(7), 1085-1093. DOI: 10.1111/j.1600-0706.2012.00070.x

Constantino, R. 2012. Insetos do Brasil: diversidade e taxonomia. Ribeirão Preto: Holos: p. 810.

Constantino, R. 2015. Cupins do Cerrado. 1 ed. Rio de Janeiro: Technical Books: p. 167.

Camargo, N. F., Ribeiro, J. F., Camargo, A. J. A., \& Vieira, E. M. 2014. Diet of the gracile mouse opossum Gracilinanusagilis (Didelphimorphia: Didelphidae) in a neotropical savanna: intraspecific variation and resource selection. Acta Theriologica, 59(1), 183-191. DOI: 10.1007/ s13364-013-0152-y
Carvalho, R. F., Passos, D. C., \& Lessa, L. G. 2019. Diet variations in short-tailed opossum Monodelphis domestica (Didelphimorphia, Didelphidae) due to seasonal and intersexual factors. Mastozoología Neotropical, 26(2), 1-9. DOI: 10.31687/saremMN.19.26.2.0.14

Dickman, A. C. R., \& Huang, C. 1988. The reliability of fecal analysis as a method for determining the diet of insectivorous mammals. Journal of Mammalogy, 69(1), 108-113.

Emmons, L. H., \& Feer, F. 1997. Neotropical rainforest mammals: a field guide. 2nd ed. Chicago: The University of Chicago Press: p. 396.

Franco, F. L., \& Ferreira, T. G. 2002. Descrição de uma nova espécie de Thamnodynastes Wagler, 1830 (Serpentes, Colubridae) do nordeste brasileiro, com comentários sobre o gênero Phyllomedusa: Journal of Herpetology, 1(2), 57-74. DOI: 10.11606/issn.2316-9079.vli2p57-74

Franco, F. L., Trevine, V. C., Montingelli, G. G., \& Zaher, H. 2017. A new species of Thamnodynastes from the open areas of central and Northeastern Brazil (Serpentes: Dipsadidae: Tachymenini). Salamandra, 53(3), 339-350.

Hammer, Ø., Harper, D. A. T., \& Ryan, P. D. 2001. PAST: Paleontological statistics software package for education and data analysis. Palaeontologia Electronica 4: 1-9. Disponível em: http://palaeo-electronica.org/2001_1/ past/issue1_01.htm.

Hurlbert, S. H. 1984. Pseudoreplication and the Design of Ecological Field Experiments. Ecological Monographs, 54(2), 187-211.

Korschgen, J. L. 1987. Procedimientos para el analisys de los hábitos alimentarios. In: Tarres, R. R. (Ed.) Manual de técnicas de Gestion de Vida Silvestre. pp. 119-134. Washington, DC: The Wildlife Society.

Leal, K. P. G., Batista, I. R., Santiago, F. L., Costa, C. G., \& Câmara, E. M. V. C. 2008. Mamíferos Registrados em três Unidades de Conservação na Serra do Espinhaço: Parque Nacional da Serra do Cipó, Parque Nacional das Sempre Vivas e Parque Estadual da Serra do RolaMoça. Sinapse Ambiental, 40-50.

Lessa, L. G., Maria, B., Costa, D. A., Rossoni, D. M., Dias, L. G., Amorim, E., Júnior, M., \& Silva, J. D. A. 2008. Mamíferos da Cadeia do 
Espinhaço: riqueza ameaças e estratégias para conservação. Megadiversidade, 4, 218-232.

Lessa, L. G., \& Costa, F. N. 2009. Food habits and seed dispersal by Thrichomys apereoides (Rodentia: Echimyidae) in a Brazilian Cerrado reserve. Mastozoologia Neotropical, 16(2), 459463.

Lessa, L. G., Geise, L., \& Costa, F. N. 2013. Effects of gut passage on the germination of seeds ingested by didelphid marsupials in a neotropical savanna. Acta Botanica Brasilica, 27(3), 519-525. DOI: 10.1590/S010233062013000300009

Lessa, L. G., \& Paula, C. S. 2014. Estrutura da comunidade de pequenos mamíferos em uma área de mata ciliar savânica no Parque Estadual do Rio Preto, Minas Gerais, Brasil. Neotropical Biology and Conservation, 9(2), 98-104. DOI: 10.4013/nbc.2014.92.04

Mattos, I. 2014. Influência na disponibilidade de recursos alimentares na dinâmica populacional de roedores em áreas de campos de murundus de um cerrado no Brasil central. Dissertação de mestrado. Instituto de Ciências Biológicas, Universidade de Brasília. p. 102.

Mantovani, W., \& Martins, F. R. 1988. Variações fenológicas das espécies do Cerrado da Reserva Biológica de Moji Guaçu, Estado de São Paulo. Acta Botanica Brasilica, 11(6), 101-112.

Martins, E. G., Bonato, V., Pinheiro, H. P., \& dos Reis, S. F. 2006. Diet of the gracile mouse opossum Gracilinanus microtarsus (Didelphimorphia: Didelphidae) in a Brazilian cerrado: patterns of food consumption and intrapopulation variation. Journal of Zoology, 269(1), 21-28. DOI: 10.1111/j.1469-7998.2006.00052.x

Melo, G. L., Santos, S. A., Sponchiado, J., Cáceres, N. C., \& Lessa, L. G. 2018. Feeding ecology of the marsupial Thylamys macrurus (Olfers 1818) (Mammalia, Didelphidae) in woodland patches of Cerrado, central-western Brazil. Mammalia, 83(1), 41-48. DOI: 10.1515/mammalia-2017-0068

Murphy, P. G., \& Lugo, A. E. 1986. Ecology of tropical dry forest. Annual Review of Ecology and Systematics, 17, 67-88. DOI: 10.1146/ annurev.es.17.110186.000435

Paglia, A. P., Fonseca, G. A. B. da, Rylands, A. B., Herrmann, G., Aguiar, L. M. S., Chiarello, A. G., Leite, Y. L. R., Costa, L. P., Siciliano, S., Kierulff, M. C. M., Mendes, S. L., Tavares, V. da C., Mittermeier, R. A., \& Patton, J. L. 2012. Lista Anotada dos Mamíferos do Brasil. $2^{\text {a }}$ ed. Arlington: Occasional Papers in Conservation Biology: p. 76.

Patton, J. L., Pardiñas, U. F. J., \& Guilhermo, E. (Orgs.). 2015. Mammals of South America, Rodentia. Vol. 2. University of Chicago Press: p. 4065.

Pinheiro, F., Diniz, I. R., Coelho, D., \& Bandeira, M. P.S. 2002. Seasonal pattern of insect abundance in the Brazilian cerrado. Austral Ecology, 27(2), 132-136. DOI: 10.1046/j.1442-9993.2002.01165.x

Pinotti, B. T., Naxara, L., \& Pardini, R. 2011. Diet and food selection by small mammals in an oldgrowth Atlantic forest of south-eastern Brazil. Studies on NeotropicalFaunaandEnvironment, 46(1), 1-9. DOI: 10.1080/01650521.2010.535250

Price, M. V. \& Jenkins, S. H. 1986. Rodents as seed consumers and dispersers. In: Murray, D. R. (Ed.), Seed Dispersal. p. 191-235. Sidney: Academic Press.

Sahley, C. T., Cervantes, K., Salas, E., Paredes, D., Pacheco, V., \& Alfonso Alonso. 2016. Primary seed dispersal by a sigmodontine rodent assemblage in a Peruvian montane forest. Journal of Tropical Ecology, 32(2), 125-134. DOI: $10.1017 / 266467416000043$

Sano, E. E., Rosa, R., Brito, J. L. S., \& Ferreira, L.G. 2007. Mapeamento de cobertura vegetal do bioma Cerrado: estratégias e resultados. Embrapa Cerrados. Planaltina: p. 33.

Silveira, F. A. O., Negreiros, D., Barbosa, N. P. U., Buisson, E., Carmo, F. F., Carstensen, D. W., Conceição,A.A., Cornelissen, T.G., Echternacht, L., Fernandes, G. W., Garcia, Q. S., Guerra, T. J., Jacobi, C. M., Lemos-Filho, J. P., Le Stradic, S., Morellato, L. P. C., Neves, F. S., Oliveira, R. S., Schaefer, C. E., Viana, P. L., \& Lambers, H. 2016. Ecology and evolution of plant diversity in the endangered campo rupestre: a neglected conservation priority. Plant and Soil, 403(1), 129-152. DOI: 10.1007/s11104-015-2637-8

Talamoni, S. A., Couto, D., Cordeiro Júnior, D. A., \& Diniz, F. M. 2008. Diet of some species of Neotropical small mammals. Mammalian Biology, 73(5), 337-341. DOI: 10.1016/j. mambio.2007.09.008

Triplehorn, C. A., \& Johnson, N. F. 2011. Estudo dos insetos. 2 ed. São Paulo: Cengage Learning: p. 809. 
Vieira, E. M., \& Briani, D. C. 2013. Short-term effects of fire on small rodents in the Brazilian Cerrado and their relation with feeding habits. International Journal of Wildland Fire, 22(8), 1063-1071. DOI: org/10.1071/WF12153

Zar, J. H. 2010. Biostatistical Analysis. 5 ed. Upper Saddle River, New Jersey: Prentice Hall: p. 94.

Submitted: 15 October 2019 Accepted: 16 January 2020

Published on line: 12 February 2020 Associate Editor: João Pedro Souza-Alves 